\title{
Neonatal Hypoxic-Ischemic Brain Injury: Apoptotic and Non- Apoptotic Cell Death
}

\author{
Qing Lu, Stephen M. Black*
}

Department of Medicine, University of Arizona, AZ USA

\section{Article Info}

\section{Article Notes}

Received: June 04, 2016

Accepted: July 05, 2016

${ }^{*}$ Correspondence:

Dr. Stephen M Black, PhD

Division of Translational and Regenerative Medicine,

Department of Medicine,

The University of Arizona, Tucson, AZ, 85719, USA

Email: steveblack@email.arizona.edu

(c) 2016 Black SM. This article is distributed under the terms of the Creative Commons Attribution 4.0 International License

\begin{abstract}
The neuronal cell death associated with perinatal asphyxia, or hypoxicischemic (HI) brain injury, plays an important role in neonatal mortality and neurodevelopment retardation. The types of cell death associated with $\mathrm{HI}$ in the brain have been classified as being either apoptotic or necrotic. Here we describe the recent discoveries of multiple non-apoptotic cell death pathways: necroptosis; ferroptosis; and autosis (autophagy). These new cell death pathways expand our understanding of the mechanisms underlying the cell death associated with perinatal asphyxia. By targeting specific regulators of these pathways, new therapies may be developed that could protect the neonatal brain from the $\mathrm{HI}$ mediated injury.
\end{abstract}

\section{Introduction}

The neuronal cell death associated with neonatal hypoxicischemic (HI) brain injury is one of the major causes of neonatal mortality and neurodevelopment retardation. The mortality of HI brain injury is $30-40 \%$. Significant neurological disorders and lifelong disabilities, such as cerebral palsy, seizures, visual impairment, mental retardation, learning impairment and epilepsy, occur in $20-40 \%$ of survivors ${ }^{1}$. Excitotoxicity, oxidative stress, as well as inflammation play important roles in the mechanism of neonatal HI brain injury. The cell death in $\mathrm{HI}$ injury, principally involves the cerebral cortex, hippocampus, cerebellar cortex, basal ganglia/thalamus, brainstem nuclei, and anterior horn cells of the spinal cord. The neuronal injury is influenced by the gestational age, cerebral blood supply, and the duration of the insult ${ }^{2}$. Investigations have shown that the types of cell death of neonatal brain injury are variable. Three types of cell death process have been commonly classified: apoptosis, necrosis and autophagy ${ }^{3,4}$. More recently three new non-apoptotic cell deaths pathways have been described: necroptosis, ferroptosis, and autosis (autophagy). In this review we will briefly describe how different types of cellular death impact ischemic brain injury in the neonate.

\section{Apoptosis}

The apoptotic pathway has been extensively documented in multiple animal models of neonatal brain injury ${ }^{5-7}$. Increased caspase- 3 activation has been identified in brain sections in children who died after experiencing $\mathrm{HI}^{8}$. As apoptosis is also involved in normal brain development ${ }^{9}$, the neonatal brain may be more 
susceptible to this cell death pathway than the adult ${ }^{10,11}$. Apoptosis is thought to account for a significant portion of the neuronal cell loss associated with neonatal HI events. For example in the CA1 region of hippocampus, apoptotic cells account for 18-, 35- and 43-\% of total cell numbers at 4-, 8- and 24-h after OGD (oxygen glucose deprivation) ${ }^{12}$. The apoptotic cell death pathway is histologically characterized by cytoplasmic condensation, nuclear pyknosis, chromatin condensation and fragmentation, cell rounding, membrane blebbing, cytoskeletal collapse, and the formation of membrane bound apoptotic bodies that are rapidly phagocytosed and digested by macrophages or neighboring cells. Oxidative stress plays an important role in induction of apoptosis after $\mathrm{HI}^{13}$. The key molecular players in the apoptotic pathway are APAF1, BAX, BAK, cytochrome c, as well as caspase-3. Mitochondrial dysfunction also plays a central role (Figure $1 \mathrm{~b}$ ). Caspase- 3 is cleaved in neurons within the ischemic core and penumbra, both early after reperfusion, and in the following hours and days.
This suggests that caspase- 3 participates in the delayed ischemic cell death associated with neonatal $\mathrm{HI}^{14}$. Selective caspase- 3 inhibition has been shown to reduce HI brain injury in neonatal rodent models ${ }^{15-17}$. However, caspase-3/mice exhibit increased vulnerability to neonatal $\mathrm{HI}$ injury ${ }^{18}$, indicating that a non-caspase- 3 dependent neuronal cell death pathway is involved or that caspase 3 also plays a protective role that is as yet undefined.

\section{Necrosis}

Despite numerous publications focusing on the apoptotic pathway, it should be emphasized that the majority of the cell death is necrosis (or unclassified types of cell death) ${ }^{19}$. Necrosis has been described as involving cytoplasmic swelling, nuclear dissolution and lysis. Necrosis is the major cell death phenotype in brain following $\mathrm{HI}$ injury in the neonatal rat. Within a few hours, the majority of neurons develop pyknotic nuclei and clear or eosinophilic perikarya. After $24 \mathrm{~h}$, these changes evolve

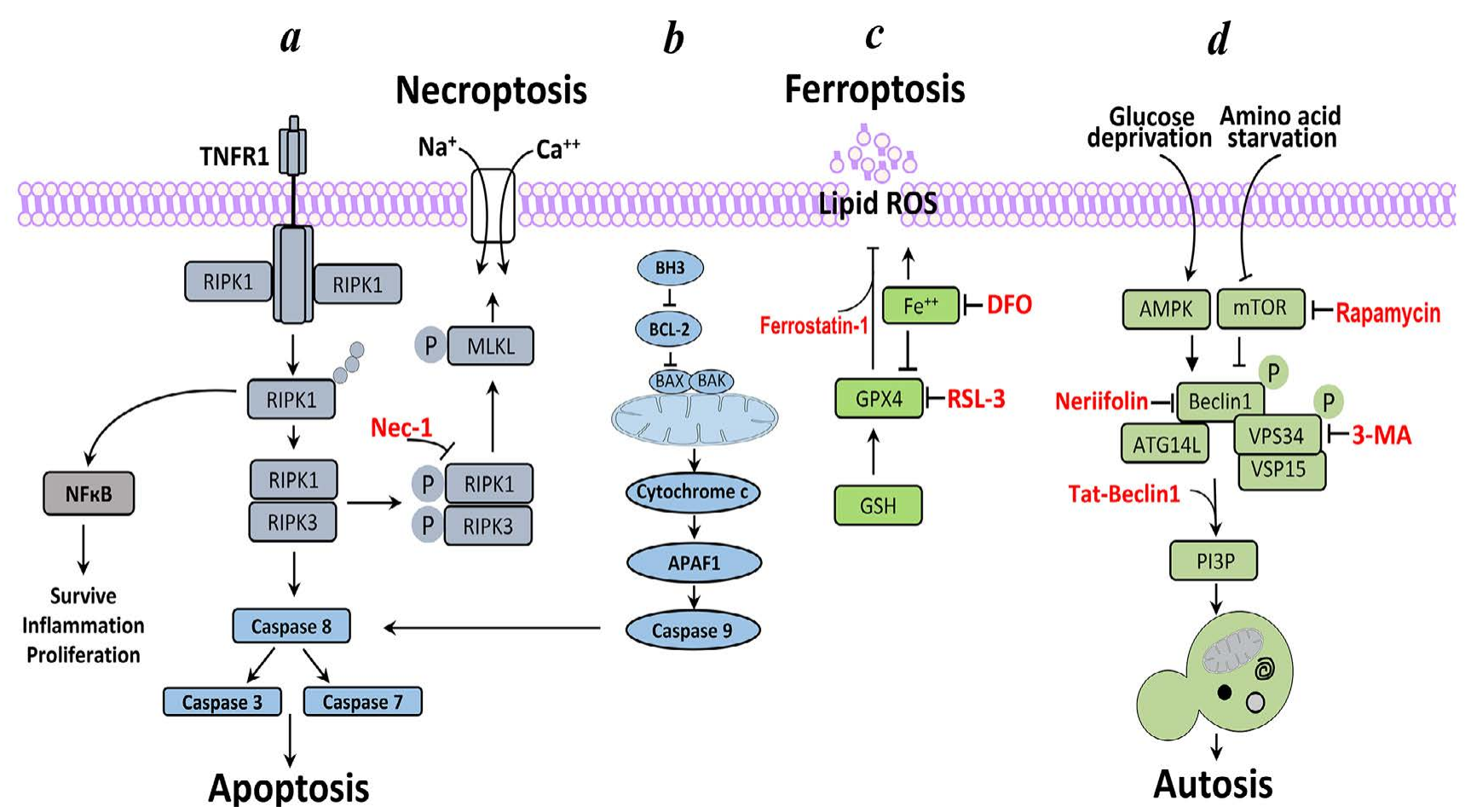

Figure 1: Neuronal cell death pathways in the neonatal hypoxic-ischemic brain injury. a. Necroptosis-Ligation of tumour necrosis factor receptor 1 (TNFR1) results in the recruitment of receptor-interacting serine/threonine protein kinase 1 (RIPK1). When caspase 8 is deleted or inhibited, RIPK1 interacts with RIPK3, to form the necrosome; this interaction can be inhibited by necrostatin 1 (NEC1). RIPK3 recruits and phosphorylates $(P)$ mixed-lineage kinase domain-like protein (MLKL), leading to the formation of MLKL oligomers that translocate to the plasma membrane. Once at the plasma membrane, MLKL forms membrane-disrupting pores, which regulate the influx of $\mathrm{Na}^{+}$and $\mathrm{Ca}^{2+}$, resulting in membrane rupture. b. Apoptosis- Cell commitment to apoptosis is regulated by the interplay between the anti-apoptotic $\mathrm{BCL}-$ 2 subfamily of proteins and the pro-apoptotic BH3-only subfamily. During apoptosis, BH3-only proteins facilitate a BAX (BCL-2-associated $X$ protein)- and BAK (BCL-2 antagonist/killer)-dependent release of cytochrome $c$, which binds to APAF1 and gives rise to the apoptosome, which, in turn, stimulates the executioner caspases 3 and 7. c. Ferroptosis-GPX4 (glutathione peroxidase-4) is the key regulator of the ferroptosis pathway, inducing iron dependent lipid peroxidation. DFO (deforxamine) an iron-chelating agent; RSL3, RAS-selective lethal compound block feroptosis. d. Autosis (autophagy)-The upstream kinases that sense cellular nutrient and energy status exert inhibitory and stimulatory effects on the Class III PI3K complex. Bcl-2 and a variety of other proteins are also involved in this step (but for simpliciaty are not presented). The Class III PI3K complex generates PI3P leading to the vesicle elongation reaction. 
into coagulation necrosis (infarction). A few days later, infarcts became partially cavitated, and by 3 -weeks a smooth-walled cystic infarct develops ${ }^{20}$. Thus, necrotic cell death has a prolonged role in the progression of brain injury and represents a prevalent type of cell death after neonatal $\mathrm{HI}^{21}$. Despite its substantial involvement in the pathological process, little effort has gone into therapeutic targeting of necrotic cell death, due to the lack of identified markers and regulatory pathways. This large gap in our knowledge regarding necrotic cell death may also be an opportunity to develop new therapeutic targets to reduce neonatal HI brain injury. Encouragingly, substantial progress has been made in recent years to broaden our knowledge of the mechanisms underlying the neuronal cell death associated with neonatal HI that hopefully will lead to new approaches to protect the neonatal brain.

Alternative path triggering cell death, to eliminate damaged or dysfunction cells, independent of apoptosis have also been discovered. Mutant mice deficient in key apoptotic factors, such as caspase or Bcl-2, can survive to adult ${ }^{22,23}$. Non-apoptotic cell death has also been observed in neonatal $\mathrm{HI}$ brain injury model ${ }^{18}$. The regulation of several non-apoptotic cell death pathways such as necroptosis, autosis, and ferroptosis have been recently reported and interest in their role in brain injury is growing rapidly.

\section{Necroptosis}

That the stimulation of Fas /TNF $\alpha$ receptor (death receptor) family triggers a canonical apoptotic pathway, including the activation of multiple caspases is well established $^{24}$. However, it has been shown that under apoptosis-deficient conditions Fas /TNF $\alpha$ can still induce non-apoptotic cell death (Fig.1 a). In this situation, TNF exposed cells were observed to undergo swelling and lysis ${ }^{25}$, with features of morphological injury that included low density vacuoles but without chromatin condensation ${ }^{26}$. More recently it has been shown that these vesicles are double membrane-enclosed, and filled with electron dense material ${ }^{27}$. The formation of these vesicles can be blocked using autophagy inhibitors. The term necroptosis is used to describe this alternative non-apoptotic cell death pathway. A specific necroptosis inhibitor, Nec-1 has been found through chemical library screening. Nec-1 efficiently inhibits $\mathrm{TNF} \alpha$-induced cell death, without the requiring caspase inhibition. Necroptosis has also been shown to occur in the injured mouse brain after middle cerebral artery occlusion (MCAO), with Nec-1 treatment significantly reducing infarct $\operatorname{size}^{27}$. Nec-1 has also been shown to improve motor function, Morris maze performance, and spatial memory in rats exposed to $\mathrm{MCAO}^{28}$. Nec-1 has also been shown to decrease injury in the forebrain and thalamus and protection correlated with decreased necrotic cell death and increased apoptotic cell death ${ }^{29}$. Nec-1 also reduces other injurious factors associated with
$\mathrm{HI}$ injury including NO generation, protein nitration, and glutathione oxidation ${ }^{30}$. More specifically, studies have suggested that the receptor-associated adaptor RIP1 is the primary cellular target of Nec- $1^{31}$. Furthermore, the death receptor is not the only activator of necroptosis. A recent study found that deletion of the acid-sensing ion channel 1a (Asic1a) gene attenuates RIP1 phosphorylation and brain injury, suggesting ASIC1a-mediated RIP1 activation is also an important pathway in ischemic neuronal cell death $^{32}$. This new form of neuronal necroptosis induced by extracellular acidosis is exciting as $\mathrm{HI}$ induces extracellular acidosis, via an increase in lactate release ${ }^{33}$, and opens up potential new avenues for both research into acid-induced neuronal cell death with the potential for new therapeutic targets being developed.

\section{Ferroptosis}

Besides necroptosis, another non-apoptotic cell death, that is iron-dependent, has recently been reported and named ferroptosis. Ferroptosis appears to be peroxidationdriven and requires abundant and accessible cellular iron (Fig.1 c). Ferroptosis was identified in cells under conditions where caspase, BAX and BAK were suppressed to attenuate apoptosis and RIP1/RIP3 expression decreased to reduce necroptosis ${ }^{34}$. Available data suggests that the lipid repair enzyme, GPX4 (glutathione peroxidase-4), is involved in this signal pathway. In the conditional ablation of GPX4 in neurons of mice, selective and rapid motor neuron degeneration is induced through ferroptosis, with no caspase-3 activation and TUNEL staining present ${ }^{35}$. Ferrostatin-1 has been identified as a small molecule inhibitor of ferroptosis, and significantly reduces the glutamate-induced cell death in mouse hippocampal slice cultures $^{36}$. NADPH oxidase generated ROS likely promotes ferroptosis cell death ${ }^{36}$. Compared to apoptosis induced by staurosporine, necrosis caused by $\mathrm{H}_{2} \mathrm{O}_{2}$ or autophagy induced by rapamycin, ferroptosis is characterized by increased mitochondrial membrane density. The role of iron in ferroptosis remains unclear. A recent study has demonstrated that hydroxyl radical generated from iron accumulation, induces neonatal cell death, through a mechanism that requires NO and Iron regulatory protein-1 (IRP-1) ${ }^{37}$. As the lipid metabolism of the developing brain is poorly understood, more investigations are needed to unravel the complex role of lipid peroxidation in neonatal HI brain injury. Recent studies have also shown that HI attenuates $\beta$-oxidation via the disruption of carnitine homeostasis and subsequently mitochondrial function ${ }^{38}$. Studies in the neonatal dog also suggest that fatty acid oxidation may contribute up to $25 \%$ of the metabolites entering the TCA cycle ${ }^{39}$. Iron is intimately involved in fatty acid oxidation, not only as a component of cytochromes within the electron transport chain, but because it is involved in the production of carnitine ${ }^{40,41}$. Further, iron 
deficiency during pregnancy may also affect neonatal brain lipid metabolism and myelination ${ }^{42}$.

\section{Autosis}

Autophagy refers to any cellular degradative pathway that involves the delivery of cytoplasmic cargo to the lysosome. It occurs at basal levels in most tissues and contributes to the protein turnover of cytoplasmic components. Adaptive autophagy exerts cytoprotective and anti-inflammatory effects ${ }^{43}$. Autophagy has been shown to be neuroprotective in some forms of acute brain damage, including methamphetamine intoxication, spinal cord injury and subarachnoid haemorrhage. However, the autophagic machinery precipitates a peculiar form of cell death (known as autosis) that appears to contribute to the development of other types of acute brain damage including neonatal asphyxia ${ }^{44}$.

Impressive progress on the autophagy-mediated cell death has been made recently. Autophagy induced cell death neither activates caspase 3 , nor is prevented by genetic deletion of RIPK1 and RIPK3, making it distinct from apoptosis and necroptosis. The term "autosis" has been coined to define the cell death mediated by autophagy genes and is characterized by focal perinuclear swelling. Autophagy (autosis) involves the degradation of cellular components via the lysosome machinery. The major intracellular characteristics of autophagy are the sequestration of damaged proteins and dysfunctional organelles into the double-membrane structure, to form the autophagosome which is decorated with the autophagic protein LC3. The autophagosome subsequently fuses with lysosomes for degradation and recycling ${ }^{45}$. Multiple proteins are required for initiation, vesicle elongation, autophagosome maturation, lysosome fusion, and degradation. Targeting the autophagic machinery could have significant impact in protecting the neonatal brain from HI-mediated cell death. Although the generation of the autophagosome is not fully understood, several key regulators have been revealed. These include mTOR, AMPK, and the PI3K complex. In the initiation phase class III PI3 kinase complex is thought perform a key role along with VPS34, VSP15, ATG14L and Beclin1(Fig.1 d). This complex generates PI3P, which determines the site at which the double membrane subsequently elongates. The Beclin1-VPS34 initiation complex is controlled through a preinitiation complex, which is activated by AMPK and inhibited by $\mathrm{mTOR}^{46}$. During glucose deprivation, MAPK can also act directly on the Beclin1-VPS34 complex, by phosphorylating Beclin1 at S91/S94, and T163 /S165 in VPS34 to induce autophagy ${ }^{47}$. Accumulating evidence has shown that autophagy is associated with neuronal cell death in both neurodegenerative disease ${ }^{48}$ and in neonatal HI brain injury. A dramatic increase in autophagosome formation, accompanied with extensive neuronal cell death, has been described in the hippocampal pyramidal region of neonatal mice exposed to $\mathrm{HI}$ and mice deficient in Atg7 (Autophagy-related protein 7) in the brain exhibit nearly complete protection against neuronal cell from death ${ }^{49}$. Intense vacuolization and autophagosome formation have been observed the hippocampus CA1 and CA3 regions in a neonatal rat $\mathrm{HI}$ brain injury model ${ }^{50}$. Autophagy protein expression and autophagosome generation have also been reported in hippocampal slice cultures exposed to $\mathrm{OGD}^{51}$. Interestingly, in the rat neonatal HI brain injury model, most of the neuronal cell death in the CA3 region appears to be through autophagy, with high numbers of autophagosomes, empty vacuoles, and focal ballooning of the perinuclear space being observed ${ }^{52}$. Importantly, significant LC3 staining, as an autophagy marker, has been found in the brains of human neonate ${ }^{53}$. Thus, accumulating evidence implicates autophagy as an important player in the neuronal cell death associated with neonatal HI injury brain. Cardiac glycosides have been identified to be potent inhibitors of autosis. One such glycoside, neriifolin blocks the increase in autophagy induced by HI in the brain, and was found to be highly protective against HI-mediated brain injury ${ }^{52}$. In addition, new autophagy activators are being developed for therapeutic purposes. For example, the increasing autophagy using fluendazole, interrupts HIV transmission ${ }^{54}$. Thus, targeting the autophagy pathway has a potential therapeutic role in the spectrum of human disease.

\section{Conclusion}

The recent discovery of multiple non-apoptotic cell death pathways has expanded our understanding of the mechanism underlying the neuronal cell death associated with neonatal HI brain injury. Compounds are already being generated from small molecules screening or using designed peptides that can active or inhibit these processes. It is hoped that by targeting specific regulators of these new pathways, new therapies will be developed that are able to protect the neonatal brain.

\section{References}

1. Juul SE, Ferriero DM. Pharmacologic neuroprotective strategies in neonatal brain injury. Clin Perinatol. 2014;41(1):119-31.

2. Swaiman KF, Ashwal S Pediatric Neurology Principles \& Practice. 1999; Mosby.

3. Northington FJ, Ferriero DM, Flock DL, Martin LJ. Delayed neurodegeneration in neonatal rat thalamus after hypoxia-ischemia is apoptosis. J Neurosci. 2001;21(6):1931-8.

4. Zille M, Farr TD, Przesdzing I, et al. Visualizing cell death in experimental focal cerebral ischemia: promises, problems, and perspectives. J Cereb Blood Flow Metab. 2012;32(2):213-31.

5. Nakajima W, Ishida A, Lange MS, et al. Apoptosis has a prolonged role in the neurodegeneration after hypoxic ischemia in the newborn rat. J Neurosci. 2000;20(21):7994-8004.

6. Manabat $\mathrm{C}$, Han $\mathrm{BH}$, Wendland $\mathrm{M}$, et al. Reperfusion differentially 
induces caspase-3 activation in ischemic core and penumbra after stroke in immature brain. Stroke. 2003;34(1):207-13.

7. Pulera MR, Adams LM, Liu H, et al. Apoptosis in a neonatal rat model of cerebral hypoxia-ischemia. Stroke. 1998;29(12):2622-30.

8. Rossiter JP, Anderson LL, Yang F, Cole GM. Caspase-3 activation and caspase-like proteolytic activity in human perinatal hypoxic-ischemic brain injury. Acta Neuropathol. 2002;103(1):66-73.

9. Raff MC, Barres BA, Burne JF, Coles HS, Ishizaki Y, Jacobson MD. Programmed cell death and the control of cell survival: lessons from the nervous system. Science. 1993;262(5134):695-700.

10. Ferriero DM, Miller SP. Imaging selective vulnerability in the developing nervous system. J Anat. 2010;217(4):429-35.

11. Cheng Y, Deshmukh M, D'costa A, et al. Caspase inhibitor affords neuroprotection with delayed administration in a rat model of neonatal hypoxic-ischemic brain injury. J Clin Invest. 1998;101(9):1992-9.

12. Nakatomi H, Kuriu T, Okabe S, et al. Regeneration of hippocampal pyramidal neurons after ischemic brain injury by recruitment of endogenous neural progenitors. Cell. 2002;110(4):429-41.

13. Chen $\mathrm{H}$, Yoshioka $\mathrm{H}$, Kim GS, et al. Oxidative stress in ischemic brain damage: mechanisms of cell death and potential molecular targets for neuroprotection. Antioxid Redox Signal. 2011;14(8):1505-17.

14. Namura S, Zhu J, Fink K, et al. Activation and cleavage of caspase-3 in apoptosis induced by experimental cerebral ischemia. J Neurosci. 1998;18(10):3659-68.

15. Han BH, Xu D, Choi J, et al. Selective, reversible caspase-3 inhibitor is neuroprotective and reveals distinct pathways of cell death after neonatal hypoxic-ischemic brain injury. J Biol Chem 2002;277(33):30128-36

16. Han BH, Demattos RB, Dugan LL, et al. Clusterin contributes to caspase3-independent brain injury following neonatal hypoxia-ischemia. Nat Med. 2001;7(3):338-43.

17. Arvin KL, Han BH, Du Y, Lin SZ, Paul SM, Holtzman DM. Minocycline markedly protects the neonatal brain against hypoxic-ischemic injury. Ann Neurol. 2002;52(1):54-61

18. West T, Atzeva M, Holtzman DM. Caspase-3 deficiency during development increases vulnerability to hypoxic-ischemic injury through caspase-3-independent pathways. Neurobiol Dis 2006;22(3):523-37.

19. Northington FJ, Chavez-Valdez R, Martin LJ. Neuronal cell death in neonatal hypoxia-ischemia. Annals of neurology. 2011; 69:743-758.

20. Towfighi J, Zec N, Yager J, Housman C, Vannucci RC. Temporal evolution of neuropathologic changes in an immature rat model of cerebral hypoxia: a light microscopic study. Acta Neuropathol. 1995;90(4):37586.

21. Carloni S, Carnevali A, Cimino M, Balduini W. Extended role of necrotic cell death after hypoxia-ischemia-induced neurodegeneration in the neonatal rat. Neurobiol Dis. 2007;27(3):354-61.

22. Lindsten T, Thompson CB. Cell death in the absence of Bax and Bak. Cell Death Differ. 2006; 13:1272-1276

23. Los M, Wesselborg S, Schulze-Osthoff K. The role of caspases in development, immunity, and apoptotic signal transduction: lessons from knockout mice. Immunity. 1999; 10:629-639

24. Wallach D, Varfolomeev EE, Malinin NL, et al. Tumor necrosis factor receptor and Fas signaling mechanisms. Annual review of immunology. 1999; $17: 331-367$

25. Vercammen D, Vandenabeele P, Beyaert R, Declercq W, Fiers W. Tumour necrosis factor-induced necrosis versus anti-Fas-induced apoptosis in L929 cells. Cytokine. 1997;9(11):801-8.

26. Kawahara A, Ohsawa Y, Matsumura H, Uchiyama Y, Nagata S. Caspase- independent cell killing by Fas-associated protein with death domain. J Cell Biol. 1998;143(5):1353-60.

27.Degterev A, Huang Z, Boyce M, et al. Chemical inhibitor of nonapoptotic cell death with therapeutic potential for ischemic brain injury. Nat Chem Biol. 2005;1(2):112-9.

28. You Z, Savitz SI, Yang J, et al. Necrostatin-1 reduces histopathology and improves functional outcome after controlled cortical impact in mice. J Cereb Blood Flow Metab. 2008;28(9):1564-73.

29. Northington FJ, Chavez-valdez R, Graham EM, Razdan S, Gauda EB, Martin LJ. Necrostatin decreases oxidative damage, inflammation, and injury after neonatal HI. J Cereb Blood Flow Metab. 2011;31(1):17889

30. Chavez-valdez R, Martin LJ, Flock DL, Northington FJ. Necrostatin-1 attenuates mitochondrial dysfunction in neurons and astrocytes following neonatal hypoxia-ischemia. Neuroscience. 2012;219:192 203.

31.Degterev A, Hitomi J, Germscheid M, et al. Identification of RIP1 kinase as a specific cellular target of necrostatins. Nat Chem Biol. 2008;4(5):313-21.

32.Wang YZ, Wang JJ, Huang Y, et al. Correction: Tissue acidosis induces neuronal necroptosis via ASIC1a channel independent of its ionic conduction. Elife. 2016;5:e14128.

33.Ljunggren B, Norberg K, Siesjö BK. Influence of tissue acidosis upon restitution of brain energy metabolism following total ischemia. Brain Res. 1974;77(2):173-86.

34. Yang WS, Stockwell BR. Synthetic lethal screening identifies compounds activating iron-dependent, nonapoptotic cell death in oncogenic-RASharboring cancer cells. Chem Biol. 2008;15(3):234-45

35.Chen L, Hambright WS, Na R, Ran Q. Ablation of the Ferroptosis Inhibitor Glutathione Peroxidase 4 in Neurons Results in Rapid Motor Neuron Degeneration and Paralysis. J Biol Chem. 2015;290(47):28097106.

36. Dixon SJ, Lemberg KM, Lamprecht MR, et al. Ferroptosis: an irondependent form of nonapoptotic cell death. Cell. 2012;149(5):106072

37.Lu Q, Harris VA, Rafikov R, Sun X, Kumar S, Black SM. Nitric oxide induces hypoxia ischemic injury in the neonatal brain via the disruption of neuronal iron metabolism. Redox Biol. 2015;6:112-21.

38. Rau TF, Lu Q Sharma S, et al. Oxygen glucose deprivation in rat hippocampal slice cultures results in alterations in carnitine homeostasis and mitochondrial dysfunction. PLoS ONE. 2012;7(9):e40881.

39. Spitzer JJ. Application of tracers in studying free fatty acid metabolism of various organs in vivo. Fed Proc. 1975;34(13):2242-5.

40. Lindstedt G. Effect of metal ions on the hydroxylation of gammabutyrobetaine to carnitine in rat liver homogenages. Biochim Biophys Acta. 1967;141(3):492-8

41. Hulse JD, Ellis SR, Henderson LM. Carnitine biosynthesis. betaHydroxylation of trimethyllysine by an alpha-ketoglutarate-dependent mitochondrial dioxygenase. J Biol Chem. 1978;253(5):1654-9.

42. Dobbing J. Iron deficiency disease in infants. 1990; Springer.

43. Kroemer G, Marino G, Levine B. Autophagy and the integrated stress response. Molecular cell. 2010; 40:280-293

44. Galluzzi L, Bravo-San Pedro JM, Blomgren K, Kroemer G. Autophagy in acute brain injury. 2016; Nat Rev Neurosci.

45. Choi AM, Ryter SW, Levine B. Autophagy in human health and disease. The New England journal of medicine. 2013; 368:651-662.

46. Green DR, Levine B.To be or not to be? How selective autophagy and cell death govern cell fate. 2014; Cell 157:65-75 
47. Kim J, Kim YC, Fang C, et al. Differential regulation of distinct Vps34 complexes by AMPK in nutrient stress and autophagy. Cell. 2013;152(12):290-303.

48. Rubinsztein DC. The roles of intracellular protein-degradation pathways in neurodegeneration. Nature. 2006;443(7113):780-6.

49. Koike M, Shibata M, Tadakoshi M, et al. Inhibition of autophagy prevents hippocampal pyramidal neuron death after hypoxic-ischemic injury. Am J Pathol. 2008; 172:454-469

50. Sheldon RA, Hall JJ, Noble LJ, Ferriero DM (2001) Delayed cell death in neonatal mouse hippocampus from hypoxia-ischemia is neither apoptotic nor necrotic. Neuroscience letters. 2001; 304:165-168
51.Lu Q Harris VA, Kumar S, Mansour HM, Black SM. Autophagy in neonatal hypoxia ischemic brain is associated with oxidative stress. Redox Biol. 2015;6:516-23.

52.Liu Y, Shoji-Kawata S, Sumpter RM, Jr., et al. Autosis is a $\mathrm{Na+,K+-}$ ATPase-regulated form of cell death triggered by autophagy-inducing peptides, starvation, and hypoxia-ischemia. Proc Natl Acad Sci U S A. 2013; 110: 20364-20371

53.Xie C, Ginet V, Sun Y, et al. (2016) Neuroprotection by selective neuronal deletion of Atg7 in neonatal brain injury. Autophagy 12:410-423.

54.Chauhan S, Ahmed Z, Bradfute SB, et al. Pharmaceutical screen identifies novel target processes for activation of autophagy with a broad translational potential. Nature communications. 2015; 6:8620 